\title{
EchoGéo
}

19 | 2012

Du littoral à la haute mer : quelles recherches récentes en géographie?

\section{Entretien avec Noël Le Scouarnec}

Maria Gravari-Barbas et Sébastien Jacquot

\section{(2) OpenEdition \\ Journals}

Édition électronique

URL : https://journals.openedition.org/echogeo/13006

DOI : 10.4000/echogeo. 13006

ISSN : 1963-1197

Éditeur

Pôle de recherche pour l'organisation et la diffusion de l'information géographique (CNRS UMR 8586)

Référence électronique

Maria Gravari-Barbas et Sébastien Jacquot, «Entretien avec Noël Le Scouarnec », EchoGéo [En ligne], 19 | 2012, mis en ligne le 10 février 2012, consulté le 10 août 2021. URL : http://

journals.openedition.org/echogeo/13006 ; DOI : https://doi.org/10.4000/echogeo.13006

Ce document a été généré automatiquement le 10 août 2021.

EchoGéo est mis à disposition selon les termes de la licence Creative Commons Attribution - Pas d'Utilisation Commerciale - Pas de Modification 4.0 International (CC BY-NC-ND) 


\title{
Entretien avec Noël Le Scouarnec
}

\author{
Maria Gravari-Barbas et Sébastien Jacquot
}

1 Noël Le Scouarnec travaille à la Direction générale de la compétitivité, de l'industrie et des services (Dgcis), Ministère de l'Économie.

2 - Maria Gravari-Barbas (MGB), Sébastien Jacquot (SJ.). Quel est votre parcours de géographe ? De quelle manière vous a-t-il amené vers votre carrière professionnelle?

3 - Noël Le Scouarnec (NLS). La géographie a toujours été une évidence pour moi, un centre d'intérêt prioritaire dès le plus jeune âge. Tout d'abord, quelques indications sur ma formation et mon parcours: doctorat de $\mathrm{III}^{\mathrm{e}}$ cycle en géographie effectué à l'Université de Paris 1 en 1980 sous la direction de Michel Rochefort sur le thème "Croissance urbaine et tourisme sur le littoral malouin", après une maitrise en géographie humaine réalisée avec Jacqueline Beaujeu-Garnier sur la "politique communale et aménagement en banlieue parisienne». Ce cursus universitaire de géographe est complété par la formation, alors pratiquement unique en France, au Centre d'Etudes Supérieures du Tourisme de Paris1.

4 A la fin de mes études, je suis recruté assez rapidement à la Direction du Tourisme, après une expérience de deux ans dans l'urbanisme au sein d'un cabinet de la Caisse des dépôts et Consignations, le BETURE. Mon expérience est très diversifiée - et durable dans cette administration du tourisme, pendant une trentaine d'années. Tout d'abord, au cours des années 70 / 80 mon champ d'activités couvre la politique sociale des vacances, et notamment le financement des villages de vacances dans leur création et leur rénovation, avec une expertise des projets dans les localités d'accueil, et ensuite dans la mise en œuvre des textes de création du chèque vacances et de l'Agence Nationale pour les Chèques Vacances.

Lors de cette période, je me consacre également à l'instruction du financement par l'État des hébergements hôteliers et de campings, en étroite relation avec la direction du Trésor, le Crédit Hôtelier / CEPME et le Crédit national. Le territoire est très présent dans cet itinéraire professionnel, lorsqu'il s'agit de contribuer à la conception et au développement d'outils d'aide au montage de projets touristiques dans le cadre des contrats de plan Etat - Régions, avec notamment la création du Fonds d'Aide au Conseil 
et à l'Innovation Touristique (FACIT). Cet instrument permet, à partir de la fin des années 1980, l'analyse de la faisabilité des projets publics et privés au plan local.

6 Au cours des années 90, j'interviens sur les politiques concertées de valorisation du patrimoine avec les ministères de la Culture et de l'Environnement (valorisation des sites culturels et naturels), et sur la modernisation des stations touristiques littorales en collaboration avec la DATAR (Délégation interministérielle à l'Aménagement du Territoire et à l'Action Régionale).

7 De même, l'opportunité m'est ensuite offerte de travailler sur les actions en faveur de la formation initiale et professionnelle et de l'emploi, puis sur le programme nouveaux services-emplois jeunes, en relation étroite avec les branches professionnelles tant au plan national, que dans les régions.

8 J'interviens également pour accompagner l'émergence du tourisme urbain en France, en appuyant les initiatives des réseaux de villes afin de contribuer à l'accroissement de leur attractivité touristique, notamment des métropoles régionales.

9 Enfin, à partir de la fin des années 90, j'anime un service de coordination et d'initiation d'études, puis de recherche, sur les différents champs d'activités et enjeux du tourisme. Depuis 2009, mon champ de compétences s'est élargi aux domaines de l'industrie, des services, du commerce, avec un accent plus particulièrement porté sur la compétitivité et le développement des entreprises au sein de la Direction Générale de la Compétitivité, de l'Industrie et des Services (DGCIS).

10 - MGB, SJ. Plus précisément, de quelle manière votre formation de géographe vous a-telle armé sur le plan conceptuel et méthodologique dans l'exercice de votre métier?

11 - NLS. A la sortie de l'université, mon thème de recherche, découlant directement de ma formation de géographe, porte sur l'urbanisme opérationnel, suivi par une première expérience professionnelle en tant que chargé d'étude junior dans l'urbanisme opérationnel au BETURE, en 1974. Il s'agit alors de réaliser des dossiers sur l'urbanisme des agglomérations de plus de 10000 habitants (évolution du logement et de l'urbanisme commercial) aboutissant à un dossier constitué de données statistiques et économiques avec de nombreuses représentations cartographiques décrivant la situation actuelle et les perspectives d'évolution à moyen et long termes. Ces dossiers sont utilisés par le SCET et la SCIC appartenant également au groupe de la Caisse des Dépôts et des Consignations (CDC).

12 La formation que j'ai suivi par ailleurs au Centre d'Etudes Supérieures du Tourisme, avec les interventions de géographes tels Louis Burnet et Georges Cazes, m'a offert la possibilité de conduire un travail de recherche sur l'urbanisation touristique sur le littoral; choix de géographe que ce thème permettant d'analyser à travers le développement de l'immobilier de loisirs, les conflits entre l'essor de l'économie touristique, la préservation de l'environnement, et le maintien des activités agricoles et maritimes. Le concept de développement durable ou soutenable existe peu au début des années 1980. Celui de gouvernance du développement par les collectivités locales émerge tout juste.

13 C'est une bonne introduction aux métiers du tourisme; mon intégration dans l'administration du tourisme m'offre ensuite la possibilité d'évoluer sur différents champs d'activité en complétant ma formation de géographe par des connaissances en gestion, en marketing, en économie en particulier. Cette faculté du géographe de 
s'adapter facilite mon ouverture à des disciplines connexes et me conduit à intervenir de plus en plus dans des métiers où l'économie joue un rôle déterminant.

14 - MGB, SJ. Estimez-vous que la pensée géographique structure toujours votre approche professionnelle?

15 - NLS. La dimension spatiale a toujours été très présente dans mon activité professionnelle, en particulier dans les activités liées au tourisme. Le territoire est un réceptacle pour la mise en place des projets qu'ils soient publics ou privés, qu'ils concernent des équipements de tourisme et de loisirs, des événements ou la conception de produits. L'approche systémique est également constamment prise en compte dans ce secteur complexe du tourisme et des loisirs.

16 Un exemple de cette approche territoriale est celui de la mise en place de programmes interministériels de formation-développement. Il s'agit, au début des années 1990, d'accompagner la conception et le déroulement de projets locaux par des formations adaptées, créées sur mesure pour le groupe d'acteurs directement concernés et pour qualifier leur action sur le terrain. Dans ce cadre, les organismes de formation sont sollicités par des structures réceptrices (Pays d'Accueil Touristique, Associations de pays, Chartes intercommunales, Communautés de communes...) pour répondre à des appels d'offre de formation prenant en compte des problématiques locales. Pour élaborer sa réponse, le centre de formation doit procéder à une analyse de la demande formulée par les acteurs du terrain. Ceci lui permet d'identifier les besoins réels de formation, qu'ils aient un caractère individuel ou collectif. Je trouve dans cette expérience qui a duré plusieurs années un cadre passionnant où viennent en convergence le territoire, l'entreprise et la ressource humaine locale.

17 Autre exemple, la mise en œuvre d'un programme national visant la modernisation et une meilleure organisation des stations sur le littoral. La méthode préconisée, consistant en la réalisation d'un diagnostic décliné en un programme pluriannuel d'actions, s'inspire largement de l'approche géographique qui intègre les systèmes complexes de jeux d'acteurs au plan local.

18 La formation de géographe m'a permis de m'approprier les dossiers car elle m'a habitué à aborder en permanence un champ très large et diversifié de situations complexes à gérer, en France ou au plan international, dans une approche systémique et non pas en tant que spécialiste ou expert d'un domaine d'activités très précis.

19 Le fait d'être constamment en situation de réaliser un état des lieux, un diagnostic des conditions de développement urbanistique et / ou touristique de collectivités locales, associées le plus souvent à des partenaires privés, a été un exercice extrêmement motivant et formateur tout au long de mon itinéraire professionnel, exercé pour l'essentiel au plan national, qui m'ont permis d'aborder les problématiques liées à la représentation des thèmes majeurs du fonctionnement du territoire. Il s'agit de s'appuyer sur l'analyse des atouts, faiblesses, opportunités et menaces (AFOM) du territoire pour optimiser les potentialités de développement touristique.

Plus récemment dans mes fonctions à la DGCIS visant à coordonner le programme d'études de la direction et les travaux de prospective, ce profil de géographe facilite l'intermédiation entre les experts des domaines économiques et technologiques, et les synergies indispensables à l'avancement des projets.

21 - MGB, SJ. En quoi votre formation de géographe est-elle importante pour le travail que vous effectuez actuellement au sein de la sous-direction du Tourisme? 
22 - NLS. Cette formation a été très importante dans l'administration chargée du Tourisme, rattachée pendant très longtemps au ministère de l'Equipement, devenu depuis quelques années le ministère de l'Ecologie et du Développement Durable. Le rattachement du tourisme au ministère de l'Économie a mis l'accent plus encore sur l'approche de l'entreprise. Ayant à intervenir dans l'animation du pôle interministériel de prospective et d'anticipation des mutations économiques (PIPAME) qui rassemble près d'une dizaine de départements ministériels, la complémentarité des approches liées au territoire, à l'entreprise et à la ressource humaine permet de satisfaire l'appétit du géographe pour la mise en situation territoriale. Néanmoins, les enjeux liés aux mutations en particulier dans l'industrie font que la préoccupation économique est permanente ; cette dimension, le géographe l'intègre aisément dès lors que l'analyse économétrique, nécessaire dans les évaluations, est confiée à des spécialistes, dans une complémentarité des profils et des métiers.

23 - MGB, SJ. Votre formation en géographie vous a-t-elle été utile dans vos activités professionnelles qui relèvent de la recherche appliquée?

24 - NLS. Au cours de la période 1997 - 2009, j'anime le service en charge des études du ministère chargé du Tourisme, étendu à la recherche à partir du début des années 2000. Il s'agit alors de créer des conditions facilitatrices pour la consolidation des réseaux de chercheurs (universitaires et laboratoires de recherche), afin de leur permettre d'intervenir dans des activités touristiques sur les différents plans d'expression (géographie, économie, sociologie, ....).

25 Ce rôle de facilitateur de projets de recherches, joué par l'administration apparaît alors nécessaire du fait de la taille réduite des équipes de chercheurs, de leur dispersion sur le territoire et de leur manque flagrant de moyens. L'administration peut, en ce début des années 2000, être initiatrice de thèmes de recherche (par exemple sur les problématiques liées au changement climatique par rapport au tourisme, ou encore sur l'impact de l'évolution des prix du foncier sur l'activité touristique en montagne ou sur le littoral).

26 La passerelle entre cet emploi et la recherche appliquée permet de développer des échanges et des rencontres au plan international, notamment avec l'Espagne, à l'exemple de l'organisation des premières rencontres franco-espagnoles sur le thème « développement des territoires et management touristique ». 50 chercheurs français et espagnols interviennent ensemble, à notre initiative, en ce mois de décembre 2004 à Arles, pour expertiser les modèles touristiques des deux pays.

27 Plus récemment, la création de la direction générale de la compétitivité, de l'industrie et des services au sein du ministère de l'Économie, fournit l'occasion d'un élargissement sensible de mes fonctions à la démarche prospective sur d'autres secteurs économiques (industrie, commerce, services ...) au-delà du tourisme, et ainsi de découvrir d'autres réseaux d'experts et de chercheurs.

28 - MGB, SJ. Peut-on dire que la pensée géographique structure la manière avec laquelle vous exercez votre métier?

29 - NLS Cette expérience de près de 35 ans dans le développement des activités touristiques, puis dans l'industrie et les services, s'appuie constamment sur les méthodes issues de la géographie: capacité de synthèse, de hiérarchisation des phénomènes, de représentation clarifiée de systèmes complexes, de démarches à visée opérationnelle à partir d'un diagnostic établi le plus rigoureusement possible, de prise 
en compte des facteurs humains dans la genèse des projets de développement locaux ... ces méthodes sont également utilisées dans les cours que je dispense sur le montage de projets touristiques ou dans les directions de travaux des étudiants.

30 C'est ce que je retiens de cette formation de géographie qui m'a profondément structurée, même si elle a été complétée et enrichie par l'influence bénéfique des disciplines sœurs connexes, tout au long de ces années d'activité professionnelle au service de la collectivité. 